\title{
Evaluation of a systematic digitized training program on the effectivity of subgingival instrumentation with curettes and sonic scalers in vitro
}

\author{
Christian Graetz $^{1}$ (1) Paula Fecke ${ }^{1}$ - Miriam Seidel ${ }^{1} \cdot$ Anne Sophie Engel $^{1} \cdot$ Susanne Schorr ${ }^{1} \cdot$ Johanna Sentker $^{1}$. \\ Christof E. Dörfer ${ }^{1}$. Sonja Sälzer ${ }^{1}$
}

Received: 21 November 2019 / Accepted: 19 May 2020 / Published online: 30 May 2020

(C) The Author(s) 2020

\begin{abstract}
Objectives Whereas the key role of subgingival instrumentation in periodontal therapy is well known, the influence of operators' experience/training with different devices on treatment results is yet uncertain. Therefore, we assessed untrained undergraduate students, working on manikins, as to how effectively they learn to use curettes (GRA) and sonic scalers (AIR); hypothesizing that AIR will result in higher relative cleaning efficacy (RCE) than GRA.

Material and methods Before baseline evaluation (T0), 30 operators (9 males, 21 females) received a 2-h theoretical lesson for both instruments, followed by a 12-week period with a weekly digitized training program for 45 min. During three sessions (T1T3), the operators had to instrument six equivalent test teeth with GRA and AIR. At T0-T3, treatment time, proportion of removed simulated biofilm (RCE-b), and hard deposits (RCE-d) were measured.

Results At T0, RCE-b was in mean(SD) 64.18(25.74) \% for GRA, 62.25(26.69) \% for AIR; $(p=0.172)$ and RCE-d 85.48(12.32) $\% / 65.71(15.27) \%(p<0.001)$. At T3, operators reached highest RCE-b in both groups (GRA/AIR 71.54(23.90) $\% / 71.75(23.05) \% ; p=0.864)$; RCE-d GRA/AIR: 84.68(16.84) \%/77.85(13.98) \%; $p<0.001)$. Both groups achieved shorter treatment times after training. At T3, using curettes was faster (GRA/AIR 16.67(3.31) min/19.80(4.52) min; $p<0.001)$.

Conclusions After systematic digitized training, untrained operators were able to clean $70 \%$ of the root surfaces with curettes and sonic scalers.

Clinical relevance It can be concluded that a systematic digitized and interactive training program in manikin heads is helpful in the training of root surface debridement.
\end{abstract}

Keywords Scaling and rootplaning $\cdot$ Non-surgical periodontal therapy $\cdot$ Subgingival hard deposits $\cdot$ Training evaluation $\cdot$ Biofilm removal

\section{Introduction}

Periodontitis is described as a multifactorial inflammatory disease associated with dysbiotic plaque biofilms and characterized by progressive destruction of the tooth-supporting apparatus [1]. Whereas untreated periodontitis eventually leads to tooth loss, an adequate periodontal therapy achieves biological compatibility

Christian Graetz

graetz@konspar.uni-kiel.de

1 Clinic for Conservative Dentistry and Periodontology, School of Dental Medicine, Christian-Albrechts-University of Kiel, Arnold-Heller-Str. 3, Haus B, 24105 Kiel, Germany of the previously diseased root surfaces [2, 3], thereby, allowing reattachment of adjacent tissues [4-7]. To do so, nonsurgical mechanical elimination of biofilm and/or hard deposits (mineralized biofilm) with, e.g., hand instruments or powered scaler [8, 9] is the cornerstone of each periodontal therapy and until now without any equivalent alternative [10]. Hence, it is the first step in a comprehensive periodontal disease treatment plan and further surgical interventions are not needed in most cases [11]. However, an effective performance of mechanical plaque removal is a prerequisite and needs to be trained intensely. Usually, before treating in clinical conditions, manual skills of dental students are trained in manikin heads. The challenge is to correctly and effectively apply appropriate instruments on a dentition. Hence, such a systematic training should include repetitive 
practical procedures [12]. Hereby, the aim is to increase the students' experience and effectiveness in removal of simulated biofilm/hard deposits as well as to minimize adverse impacts [13]. Although the artificial models do not simulate a perfect realistic situation, the training is an important step to shorten treatment time and improve results [14]. Furthermore, a direct visualization of the percentage of biofilm and hard deposit removal is only possible in an in vitro situation.

Therefore, the aim of the study was to assess the training effect of a new digitally supported education program with regard to efficacy as well as time for removal of simulated biofilm and of hard deposits, with different instruments on untrained undergraduate students. Our main hypothesis was that the training effect for sonic scalers (AIR) compared with curettes (GRA) is greater at the end of the observation time, and the effect will be determined by specific factors, e.g., tooth type or jaw.

\section{Material and methods}

\section{Experimental setup}

All operators had the same setup and options to choose their instruments for root surface instrumentation; (1) Gracey normal-shape curettes nos. 5/6, 7/8, 11/12, and 13/14 (American Eagle Instruments, Missoula, MT, USA) and (2) sonic scaler (Synea, W\&H, Bürmoos, Austria) with air pressure and water-cooling $(30 \mathrm{ml} / \mathrm{min})$ as recommended by the manufacturer on level two, which means "medium" amplitude, combined with a straight, right and left curved slimline tip (1 AP, 2APr, 2APl, W\&H, Bürmoos, Austria) with round cross-section.

New instruments were used only at baseline (T0), for following visits the curettes were randomized to each operator, whereby prior to each test day (T1-T3), the curettes were controlled by a trained dental hygienist regarding sharpness and if necessary sharpened with an Arkansas stone and grinding oil. Similarly, each tip for the sonic device was controlled for their length as advised by the manufacturer or any possible destruction.

\section{Operators training program and instrumentation procedure}

All participants received the same training program including two lectures of theoretical application information according to our clinical and the manufacture's guideline as well as practical sessions in vitro before baseline test (Fig. 1). Afterwards, both groups of instruments were trained equivalently 10 times for 45 min over a 14 -week period by using a digitally modified learning program (for AIR/GRA 24/32 working steps) based on a program introduced in 1994 [12]. Concomitant to the digitized training program the group was supervised and monitored through the education staff ( 3 board certified specialist of periodontology). In a previously published study [15], we could demonstrate that training of root surface instrumentation should not only focus on efficacy, but also on ergonomics. The digitized visualization program is supposed to support the teaching of the necessary working steps and ergonomic aspects separately for each instrument, and concomitantly reduces time effort for supervision by the staff. Partly by animated GIF or short video sequences the whole set up, technique and sitting position of the operator and patient are explained. Additionally, all participants were during training sessions clinically calibrated regarding the application pressure $(3-5 \mathrm{~N}$ for GRA and $<1 \mathrm{~N}$ for AIR), but no measuring of the root surface destruction or roughness were done at the test days.

Before the study, at the beginning of the workshop all students of the 7th semester $(n=31)$ were asked to participate in the study, and after their written consent $(n=30)$, they received a 2 -h theoretical introduction for both instruments and to the training program, but without practical exercises. One student, refused to take part in the study.

Participants were evaluated at baseline (T0) and between the training weeks at week 6 (T1), week 11 (T2) and week 14 (T3) while instrumenting 12 comparable test teeth with GRA (tooth 11, 14, 16, 31, 37, 45) and with AIR (tooth 21, 25, 17, $34,36,43)$. The instrument to begin with was randomized (Microsoft Excel 16, Microsoft Corporation, One Microsoft Way Redmond, WA, USA) for each test day and participant. Participants were asked to instrument the test teeth until they achieved subjective maximal elimination of simulated hard and soft deposits. Time for treating the predetermined six teeth per instrument was recorded for each participant.

\section{Manikin heads and test teeth}

Both groups of instruments were performed in similar manikin heads, equipped with modified periodontitis models (Frasaco, Tettnang, Germany), exhibiting pronounced periodontitis with moderate to advanced horizontal bone loss, isolated and deep vertical pockets, resulting in teeth being differently difficult to instrument regarding both anatomy and accessibility. Mean (SD) pocket probing depth (PPD) was $5.8(2.1) \mathrm{mm}$ (range, $3-11 \mathrm{~mm}$ ), separated for test teeth in GRA group (5.69 (2.01) mm (range, 3-9 mm)) versus AIRgroup (5.67 (2.22) mm (range, 3-9 mm)). Gingival masks were fixed that no operator was able to lift the mask during the test trials.

Twelve teeth and seven single- and five multi-rooted teeth, were coated with a thin layer of transparent fluorescent varnish (Shiny White-Rival de Loop Young, Berlin, Germany) between the artificial cemento-enamel junction and the alveolar bone in order to simulate adhering plaque. Coating was performed by a reproducible and standardized procedure of dipping to provide similar thickness of the applied varnish [16]. The used varnish fluoresced with bright blue color when 


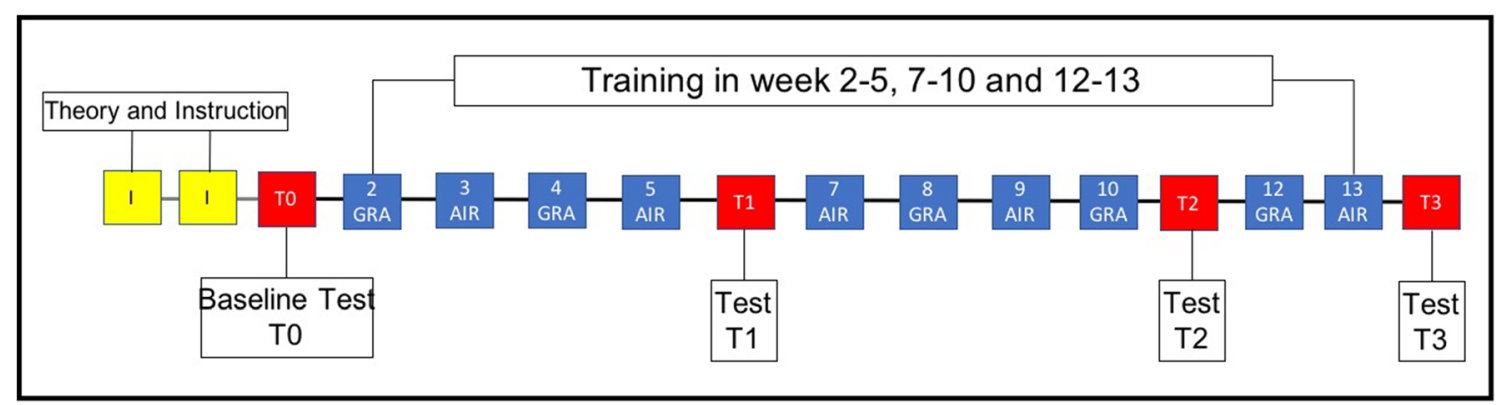

Fig. 1 Training and test protocol: All operators trained curettes (GRA) and sonic scaler (AIR) 10 times in 14 weeks in a digitized learning program. At the outset of the study (before T0), operators received

exposed to ultraviolet light. Gingival masks of persistently pliable silicon (Frasaco, Tettnang, Germany) were used to cover the models to ascertain instrumentation without visual control. Beside this simulation of biofilm, test-teeth were also randomly coated with commercial varnish (A-CK, Frasaco, Tettnang, Germany), modified for the ratio of varnish/thickener, to simulate adhering subgingival hard deposits. The hard deposits were located in spot size (diameter around $1.5 \mathrm{~mm}$ ) at each tooth type at the same area by one investigator (P.F.) according to pictures taken during the first test. The varnish on the root surfaces was placed at a distance of at least $2 \mathrm{~mm}$ from the bottom of the pocket and $1 \mathrm{~mm}$ subgingival from the marginal gingiva, to simulate the pathologic circumstances as realistic as possible.

Artificial biofilm and hard deposits as well as teeth and gingival masks were renewed after each test day (T0-T3).

\section{Planimetric evaluation}

After each test day $\mathrm{T} 0-\mathrm{T} 3$, the effectivity of instrumentation was planimetrically assessed. Instrumented teeth were mounted on standardized prepared plastic units (Lego $\mathrm{GmbH}$, Grasbrunn, Germany), individually fitting to each of the 12 teeth. Plastic units with mounted teeth were then fixed on the camera table in a reproducible position. Following, teeth were submitted to ultraviolet light (UV-A, 350-370 $\mathrm{nm}$ ), and one image of each of the four tooth surfaces (mesial, distal, vestibular, oral) (in total 1728) was taken using a camera with a $100-\mathrm{mm}$ macro-zoom (Canon EOS D30, Tokyo, Japan). The images were used to subsequently perform an evaluation of the cleaned surface area by digital image subtraction (Image J, NIH, Bethesda, USA) to calculate the relative cleaning efficacy of simulated biofilm removal subgingival (RCE-b in \%) (Fig. 2). For multi-rooted teeth with furcation involvement $(16,37,17,36)$, no evaluation of the furcation area was done (please see Fig. $2 \mathrm{c}$ for details). The relative cleaning efficacy of hard deposits (RCE-d) was assessed through one investigator (P.F.) by counting the non-removed hard deposits on reproducible images of each tooth site. instructions and handling information (I) of the instruments in two lectures. Test teeth were evaluated at baseline (T0) and between the training weeks (T1, T2, and T3)

\section{Outcomes}

As the primary outcome, the percentage of removed artificial biofilm and hard deposits were determined. Secondary outcomes were treatment time, difference between test days and instruments according to the cleaning effectiveness as well as operators' experience reports.

\section{Relative cleaning efficacy of simulated biofilm removal-RCE-b in\%}

Measuring RCE as the difference of the area with simulated biofilm before and after cleaning the four different root surface areas of each test tooth ( $\mathrm{n}=48$ of 12 test teeth) and each operator $(n=30)$, separately for the two types of instrumentation (GRA vs. AIR) and the four test days (T0-T3).

\section{Relative cleaning efficacy of hard deposit removal-RCE-d in\%}

Measuring RCE-d as the difference of the number of simulated hard deposits before and after cleaning root surface areas of all test teeth $(n=12)$ and operators $(n=30)$, separately for the two types of instrumentation (GRA versus AIR) and the four test days (T0 T3). It should be noted in comparison to RCE-b, for RCE-d only the percentage of the number of removed hard deposits compared with the total number of deposits was evaluated. It was no evaluation undertaken to measure the size of the cleaned area as hard deposits were only simulated in a small spot.

\section{Treatment time in min}

Accordingly, time for treating test teeth $(n=6)$ per group of instruments (GRA versus AIR) was measured separately for each operator $(n=30)$ at the four test days $(T 0-T 3)$. Duration for changing instruments was taken into consideration.

The investigator (J.S.) was blinded to the used instruments and test days. For cleaning efficacy, two levels were defined. For level one, $\geq 70 \%$ of simulated biofilm or hard deposits had 


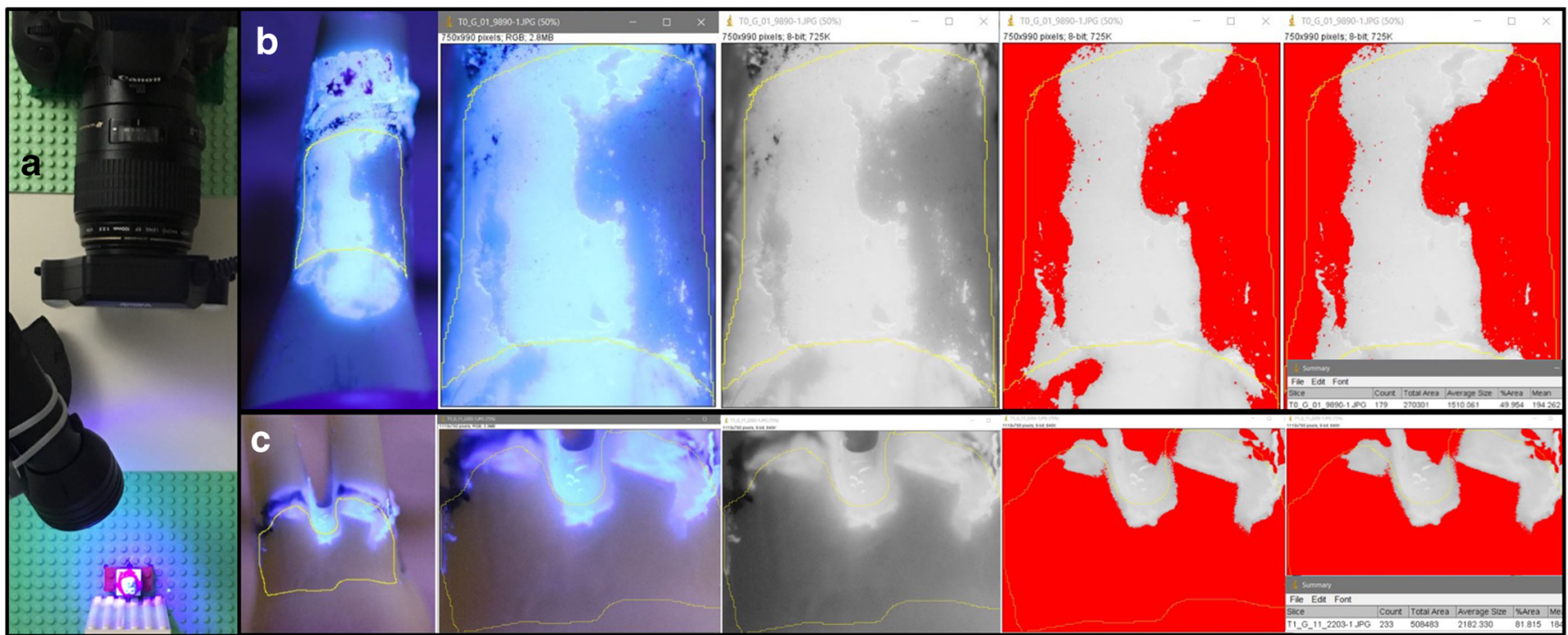

Fig. 2 a Setup for photographic documentation. The test teeth were reproducibly fixed in the test blocks in order to photograph the cleaned root surface. $\mathbf{b}$ Standardized photographic documentation exemplified for tooth 11 of the buccal root surface and $\mathbf{c}$ the oral root surface of tooth 37 .

to be removed, for level two $\geq 80 \%$. Not adequate was set as 0 $69 \%$ of removed simulated biofilm or hard deposits.

\section{Questionnaire}

At each test day (T0 - T3), operators answered a questionnaire to estimate their own effectiveness in removing simulated biofilm/hard deposits for each group of instruments as well as questions according gender, age and previous vocational training only at T0 (closed-ended question).

\section{Statistical analysis}

According to the sample size calculation with data of a comparable investigation [12] we found $n=175$ teeth per group at each test day as sufficient to detect under five percent difference for removed hard deposits between the groups of instruments (power of $80 \%$ ).

Randomization, means, percentages and standard deviations were calculated using Microsoft Excel (Microsoft Excel 16, Microsoft Corporation, One Microsoft Way Redmond, WA, USA). Values were mostly ordinal (yes/no) and with the use of Microsoft Excel, crosstabs have been drawn up. Data were entered into SPSS Statistics (SPSS Statistics 24, IBM, Chicago, IL, USA). Normal distribution of artificial biofilm, residual hard deposits and treatment time were tested by Kolmogorov-Smirnov, Shapiro-Wilk as well as Chi Quadrat Test. The artificial biofilm at the two test days (T2 and T3) was not normally distributed ( $p>0.0001)$. Artificial biofilm removal, therefore, were tested with nonparametric tests. All tests were two-sided; statistical significance was assumed $p \leq 0.05$ and adjusted with the Bonferroni
Subsequently the cleaned surfaces were evaluated in percent by a digital image subtraction (Image J, NIH, Bethesda, USA) in order to calculate the relative cleaning efficacy of simulated biofilm (RCE-b in \%)

method for multiple comparison $(\mathrm{p}=0.05 / 6=0.0083)$. Associations between variables were analyzed using Pearson correlation and Spearman's rank correlation coefficient analysis.

\section{Results}

\section{Operators' characteristics}

All 30 operators were in the seventh semester at Kiel dental school (female/male 21/9) and in mean (SD) 25.50(4.11) years of age (range 21-42). Eleven had a previous education in dentistry e.g. dental assistants or technician (female/male 7/4).

\section{Removal of simulated biofilm and hard deposits-efficacy and learning curve}

In general, the learning curve of the yet-untrained operators begins with a nearly equal efficacy level for GRA of 63.18(25.74) \% and for AIR of 62.25(26.69) \% RCE-b at T0 $(p=0.172)$. In the GRA-group RCE-b decreases until T1 to $58.62(24.18) \%(p<0.001)$ and subsequently improves significantly at T2 (RCE-b 64.05(24.51) \%, $p<0.001)$ and reaches highest efficacy at T3 (RCE-b 71.54(23.90) \%, $p<$ 0.001) (Table 1, Fig. 3). For AIR-group, we found a different performance with continually improvement of the RCE-b values after $\mathrm{T} 0$ until $\mathrm{T} 3$. At $\mathrm{T} 1$, the operators showed a $63.56(24.55) \%$ efficacy without significant changes between $\mathrm{T} 0$ and T1 $(p=0.203)$, whereas at T2 $(69.17(23.55) \%$ and T3 (71.75(23.05) \% efficacy significantly improved (T1-T2: $p<$ 0.001 and T2-T3: $p=0.001$ ) (Table 1, Fig. 3). Despite the 


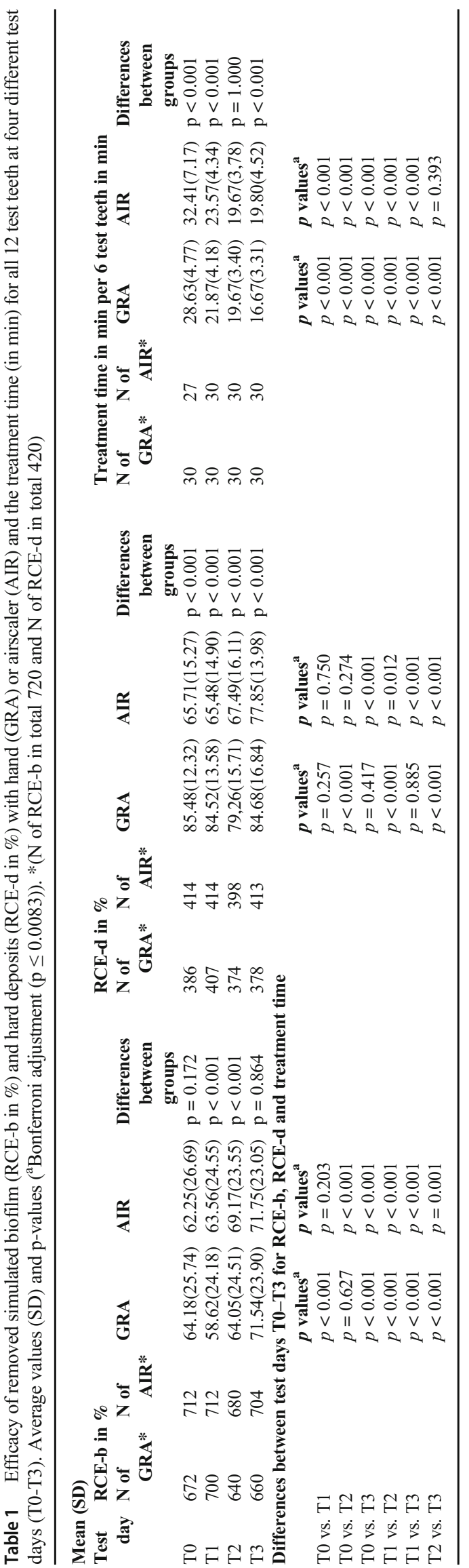

different progression during the training phase, all operators removed simulated biofilm without significant difference between both groups of instruments at the final evaluation (T3: $p$ $=0.864)$.

Considering hard deposits (RCE-d), the learning curves in the AIR-group are comparable with RCE-b. The comparison of RCE-d of both groups of instruments demonstrates significant superiority of the GRA-group at all time points (T0: GRA 85.48(12.32) \% versus AIR 65.71(15.27) \% efficacy; $p<0.001$ and T3: GRA 84.68(16.84) \% versus AIR 77.85(13.98) \% efficacy; $p<0.001)$. RCE-d values for AIR at T0 and T1/T2 (65.48(14.90) \%, $p=0.750 / 67.49(16.11) \%$, $p=0.012$ (adjusted to Bonferroni)) do not differ significantly. The improvement between $\mathrm{T} 0$ and $\mathrm{T} 3$ for RCE-d in the AIRgroup is significant $((77.85(13.98) \%, p<0.001)$ (Table 1$)$. The learning performance for GRA had a different pattern; after equal efficacy at T0 to T1 $(84.52(13.58) \%, p=0.257)$ the lowest efficacy was found at T2 (79.26(15.71) \%, $p<$ $0.001)$ and increased again until T3 (84.68(16.84) \%, $p<$ $0.001)$. The difference between $\mathrm{T} 0$ and $\mathrm{T} 3$, however, was not significant $(p=0.417)$ (Table 1, Fig. 3).

Before the beginning of the training phase, for both groups of instrument no significant difference for removing simulated biofilm in the upper jaw versus the lower jaw were measured (GRA/AIR: $p=0.011$ (adjusted to Bonferroni) $/ p=0.367$ ) (Table 2). At T3, both groups of instruments were able to reach a significant higher efficacy in the lower (GRA/AIR 68.88(23.36) \%/70.05(23.18) \%) versus upper jaw (GRA/ AIR 74.23(24.18) \%/AIR 73.39(22.38) \%) (all groups T0 T3: $p \leq 0.001)$ without significant differences between the groups of instruments (upper jaw/lower jaw: $p=0.514 / p=$ 0.638). For removal of hard deposits at $\mathrm{T} 3$ we found compared with RCE-b in the AIR-group, a higher efficacy in the upper jaw versus lower jaw (83.68(19.76) \%/74.26(12.11) \%, $p<0.001)$, but for GRA, results were nearly similar (95.95(11.52) \%/(93.14(11.28) \%, $p=0.311)$. Contrary to the training effect in simulated biofilm removal in both groups, an improvement in hard deposit removal was only observed for AIR.

On tooth-level, at T3 in the AIR group, most simulated biofilm was removed in premolars compared to molars and front teeth $(p<0.001)$, but there are no such differences for the GRA group in RCE-b (Table 2). Using GRA in molars lead to significant better RCE-b results $(p=0.001)$ compared with AIR, whereas in premolars efficacy was higher in the AIR group (81.28(19.0) \%; GRA 71.35(22.09); $p<0.001$ ).

\section{Removal of simulated biofilm and hard deposits-treatment time}

With only one exception at T2 $(p=1.000)$, the treatment time for the six teeth per group was always shorter with GRA 


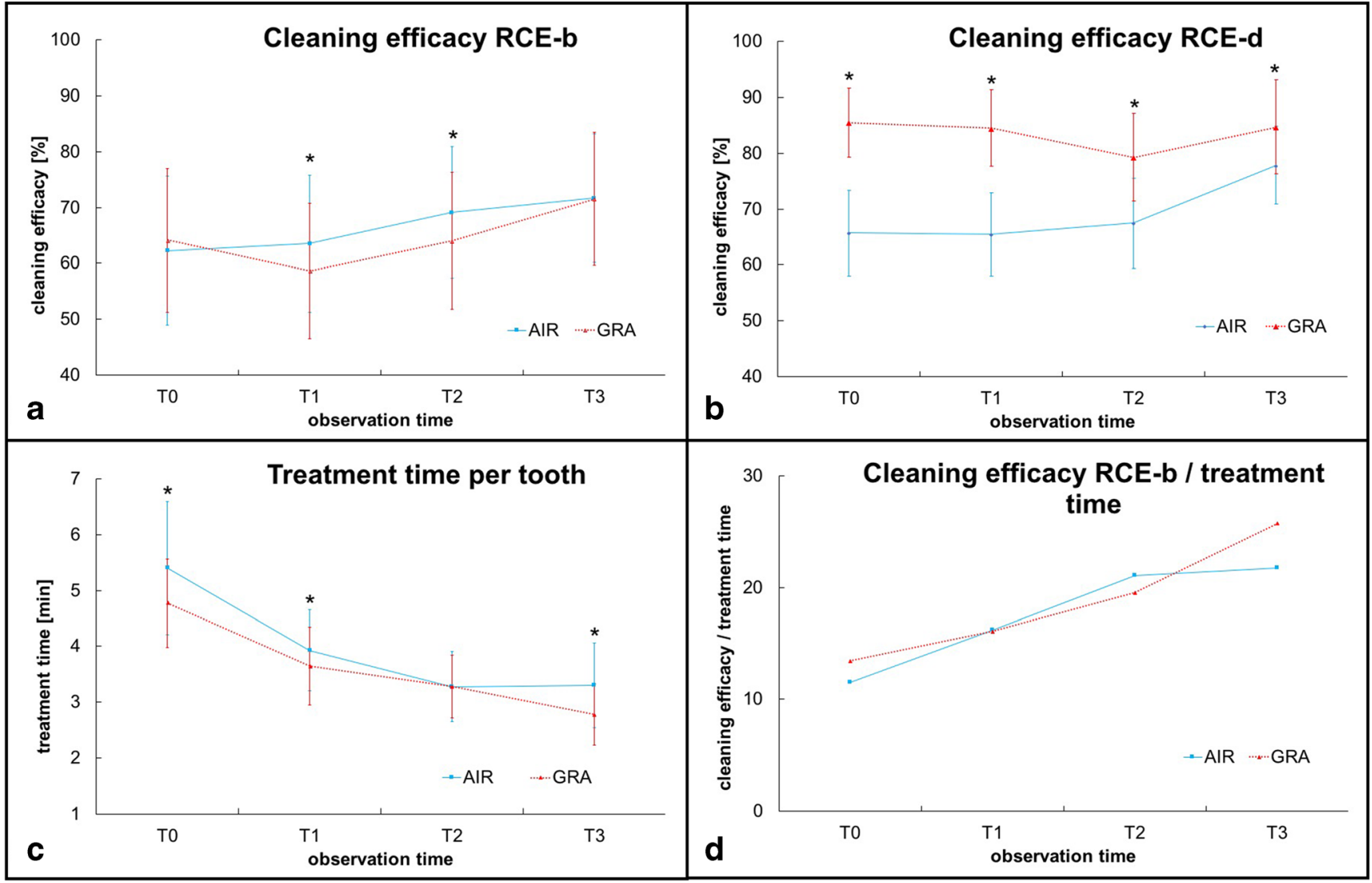

Fig. 3 Cleaning efficacy a for removal of simulated biofilm (RCE-b in $\%$ ), b removal of hard deposits (RCE-d in \%), $\mathbf{c}$ the treatment time per tooth (in $\mathrm{min}$ ), and $\mathbf{d}$ the calculated cleaning performance (RCE-b/ treatment time per tooth) for sonic instruments (AIR) versus curettes (GRA) at T0-T3. $\left({ }^{*} p \leq 0.05\right)$

learning curve was similar. Accordingly, at T0, no proband in the AIR group was able to remove all hard deposits (GRA $26.67 \%$ ), but $10 \%$ at T3 (GRA $36.67 \%$ ). Further changes in individual efficacy levels of RCE-b and RCE-d are shown in Fig. 4.

Furthermore, 18 operators showed higher efficacy to remove simulated biofilm with curettes at T3 versus T0 (AIR 24 operator) and 12 operators a lower efficacy (AIR 6 operators), respectively. For removal of hard deposits, 13 operators in the GRA group improved their own efficacy (AIR 22 operators), and 12 operators deteriorated (AIR 6 operators). Five operators showed similar results at $\mathrm{T} 0$ and $\mathrm{T} 3$ for RCE-d (AIR 2 operators).

At all test days, the operators self-estimated their efficacy of simulated biofilm removal (RCE-b) in both groups of instruments equally high with 60\%-70\% (Table 3 ).

\section{Discussion}

In a consensus report of the 1st European Workshop on Periodontal Education [13] for the undergraduate, dental 


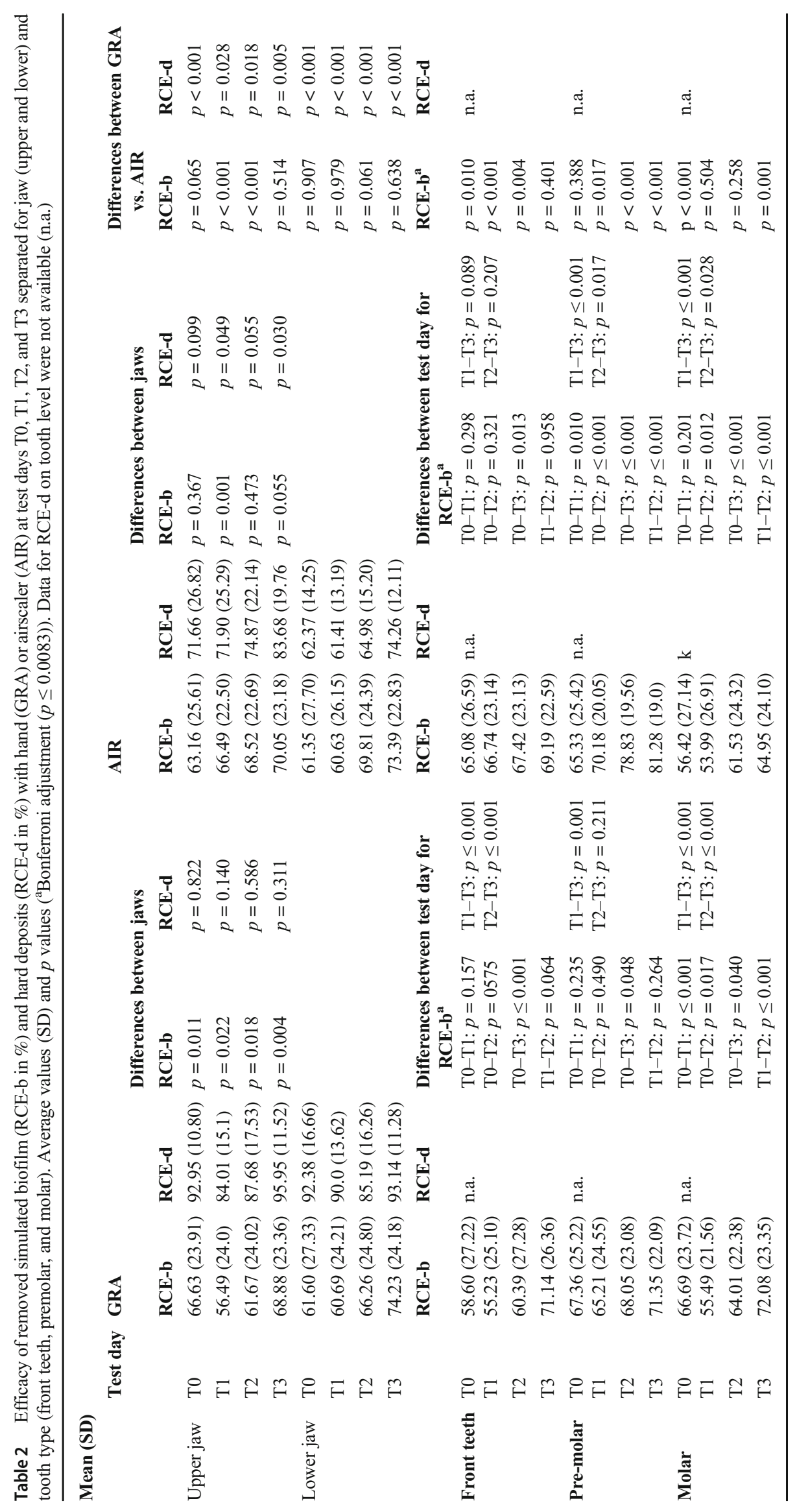


curriculum and under supporting competences is recommended that dentists must be competent in " ... undertaking supragingival and sub-gingival scaling and root surface debridement, using both powered and manual instrumentation including stain removal and prophylactic procedures ...". According to the common three levels of competence-based curricula, this corresponds to the highest level, as students should have a " ... theoretical knowledge and understanding of the subject together with an adequate clinical experience to be able to resolve clinical problems encountered, independently or without assistance ..." [13]. Although the necessity of theoretical and practical training within the framework of curricular education is indisputable, the key question remains on how to gain adequate clinical experience.

We could demonstrate in the current investigation that after a theoretical demonstration alone, untrained students were able to reach an efficacy to remove subgingival simulated biofilm (RCE-b) in mean of 64\% (GRA) or $62 \%$ (AIR), and after 6 months of training once a week for 45 Minutes, they reached $71.5 \%$ (GRA) and $72 \%$ (AIR), corresponding to a significant improvement of $7.5 \% / 10 \%(p \leq 0.001)$. Interestingly, at $\mathrm{T} 0,40 \%$ of all 30 students already achieved an efficacy of over $70 \%$ for RCE-b with curette versus $27 \%$ only with AIR. During the training phase, more than half of all operators could improve their own efficacy to remove simulated biofilm. Though, more operators were able to reach this improvement by sonic scaler (GRA/AIR 18/24 operators).

For removal of hard deposits, we found only 13 operators, which improved their own efficacy using curettes between T0 and T3 versus 22 operators using sonic scalers. The observed superior efficacy at the beginning using GRA and the concomitant lack of improvement (T0 vs T3 for GRA $85.5 \%$ vs. $85 \% ; p=0.417$ ) as well as the lower amount of operators demonstrating an improvement might be due to a structured training program on manikin heads teaching the application of a universal curette/scaler half a year before. This knowledge might now be transferred to the application of special curettes. On the other hand, they were totally inexperienced and not familiar with powered devices. Previous studies on undergraduate students, less-experienced or even experienced operators observed a similar phenomenon already $[12,16]$.

It does not seem to be surprising that the evaluated undergraduate students spend much more time for root surface debridement per tooth at the beginning of the training program (Table 2). Removal of invisible biofilm (subgingival) is difficult for less-experienced operator as they are yet not well trained in their tactility profound knowledge of the complex root morphology is missing. This leads to a significant increase of the results for RCE-b in the AIR-group already after a short time (T0-T1) and with a time delay also in the GRAgroup (T0-T2). The time delay for GRA might be explicable by the more complex handling.
At T3, we found $85 \%$ of all operators achieved an efficacy of $70 \%$ for RCE-b (AIR 87\%). For RCE-b, we failed to show significant differences between both groups of instruments before (T0) and after training (T3).

\section{Treatment time}

Contrary to older position paper, in which powered devices were often described as more effective while, e.g., saving treatment time compared with hand instruments [17] which could not be supported by the results of our study. However, differences between a manikin model and a real clinical situation should be kept in mind. Untrained operators needed in the beginning of their systematic training phase independent of the instrument used nearly five Minutes per tooth improving to about $3 \mathrm{~min}$ at T3, favoring GRA at both time points (Table $1, p<0.001$ ). Therefore, we have to reject one part of our hypothesis that less-experienced operators after 6 months of training with sonic scaler will neither reach a higher efficacy nor save treatment time compared with curettes. We can only hypothesize on these differences. In the present study, the amplitude for the sonic scaler was fixed at medium level as advised by the manufacturer, but power adjustment of powered devices will influence the results significantly [18, 19]. However, these findings are in line with previous investigations $[12,16]$. Our research underlines the recommendation for a comprehensively training of all powered devices, whose often times supposed perceived as simpler than that for hand instruments [20].

\section{Self-estimation}

The operators of the current study could, however, estimate their own efficacy and treatment time more realistically compared with previous investigations for both instruments [21]. The training took the same amount of time, but our digitized training was supported by less supervisors (here with a ratio of 1:10). Nevertheless, the influence of motivation for the training seems to be enormous [21]. Motivated students (e.g., prizes for good performance, atmosphere of competition and enthusiasm during the whole training program) can achieve about $25 \%$ higher efficacy in removal of simulated biofilm compared with a group without motivational incentives [21]. Furthermore, motivation helped to estimate the efficacy more precisely and will greatly influence learning of effective root debridement [21]. Future studies could evaluate further developed digitized training programs with, e.g., personalized digital animation and immediate feedback function.

Furthermore, magnifying loupes might be of major importance already during training. They increase visual acuity and can therefore improve clinical outcomes in dentistry [22-24]. Despite the potential advantages in wearing loupes, in the current investigation their use 

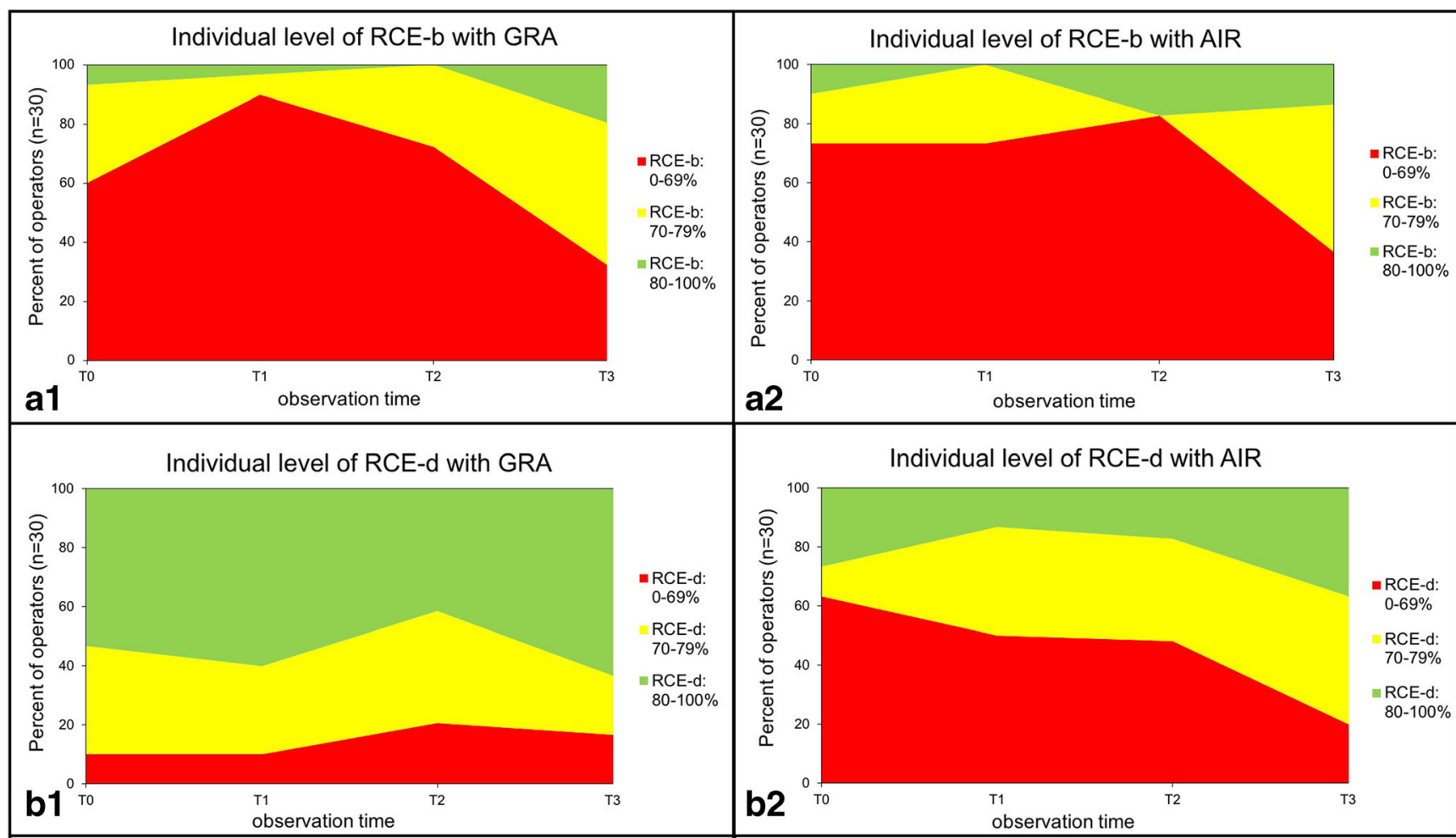

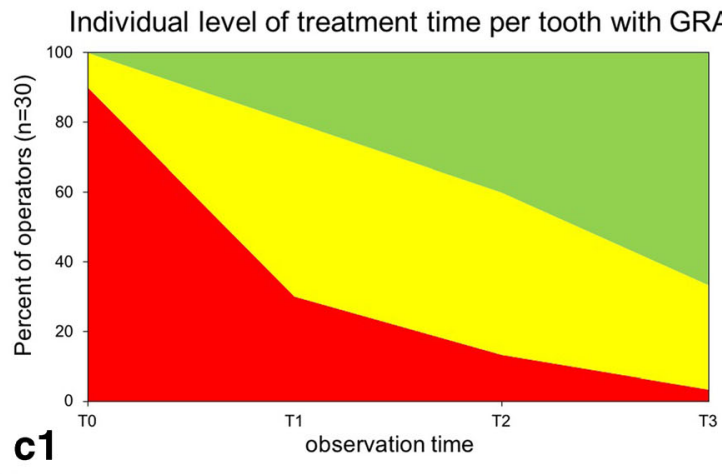

Fig. 4 a Illustration of the number of operators (left side for gracey curettes (GRA) and right side for sonic scaler (AIR) per test day with cleaning efficacy of simulated biofilm removal (RCE-b) of 0-69\%, 70$79 \%$, and $80-100 \%$ and $\mathbf{b}$ for the efficacy of hard deposit removal (RCE-

was allowed, but not obligatory. However, the benefit of loupes during periodontal treatments is controversially discussed in literature [23-27], although a better view into the sulcus can be assumed.
Table 3: Average values (SD) of efficacy of removed simulated biofilm (RCE-b) and selfestimated (in \%) by the operators for Gracey curettes (GRA) and for sonic scaler (AIR).

\begin{tabular}{|c|c|c|c|c|}
\hline & \multicolumn{2}{|l|}{ GRA } & \multicolumn{2}{|l|}{ AIR } \\
\hline & RCE-b & $\begin{array}{l}\text { Self-estimated RCE-b } \\
\text { after }\end{array}$ & RCE-b & Self-estimated RCE-b \\
\hline T0 & $64.18(25.74)$ & $70.0(9.88)$ & $62.25(26.69)$ & $64.79(10.52)$ \\
\hline $\mathrm{T} 1$ & $58.62(24.18)$ & $65.3(10.20)$ & $63.56(24.55)$ & $65.21(8.94)$ \\
\hline $\mathrm{T} 2$ & $64.05(24.51)$ & $67.50(8.14)$ & $69.17(23.55)$ & $63.33(10.80)$ \\
\hline $\mathrm{T} 3$ & $71.54(23.90)$ & $72.08(11.04)$ & $71.75(23.05)$ & $69.17(11.06)$ \\
\hline
\end{tabular}




\section{Limitations}

For successful periodontal therapy, a major goal besides effective removal of biofilm and hard deposits is not to damage the root surface [28]. This outcome was however not assessed in the present study. According to our previous in vitro investigations focusing solely on simulated biofilm $[15,16]$ or on hard deposits [27], it might be helpful to consider the goal of the treatment for unexperienced operators. Thus, given the great variety of scope of interest and study designs, all comparisons between studies require caution [16, 19, 28-34]. Further on, due to the character of the in vitro simulation, e.g., using varnish to simulate the subgingival biofilm and hard deposits on the root surface of plastic teeth does not allow transferring our findings to clinical settings in general [29]. Using varnish to simulate subgingival biofilm and hard deposits allowed reproducible and standardized conditions in vitro, but the location of artificial biofilm under the simulated hard deposits as done in the current investigation is not comparably with a clinical situation. In vivo the development of hard deposits is a dynamic process that starts with a non-mineralized biofilm, which eventually calcifies. Nevertheless, it can also be seen that it does not matter whether there is a biofilm under or above a hard deposit as both have to be removed. Complete removal of hard deposits inevitably correlates with simultaneous removal of biofilm. Further, we failed to sub-analyze results of removed simulate hard deposits on jaw/tooth level, as we calculated only the percentage of the number of removed deposits compared with the total number of deposits due to technical reasons. Also, we cannot quantify possible effects of the used gingival masks, with a possible limited penetration of curettes into the sulcus and also possible damping of oscillating instruments $[12,16,29,35]$. Furthermore, the morphology of the jaw and the tooth type are likely to have influenced our results as mentioned before. The furcation area of the involved molars was not assessed in this study, which might explain, why AIR failed to show better cleaning efficacy in molars as we assumed [17], whereas another in vitro investigation showed good accessibility when the horizontal pocket depth was less than $2 \mathrm{~mm}$ for both type of instruments [36]. Furthermore, as the tests were performed within one periodontitis model, the test teeth were not exactly corresponding but much with regard to type of tooth (front teeth, premolar, molar) in the upper and lower jaw and the profile of the used periodontitis models for both groups of instruments (AIR all quadrants vs. GRA: right upper and both lower quadrants). Therefore, we failed to control for possible confounder of handedness [37]. The analyzed teeth were selected to be comparable in terms of position (tooth type) and simulated probing depth/loss of attachment. Although, there is a difference in the location of the teeth between the two groups of instruments for premolars (teeth 14, 25 in the upper jaw and 34,45 in the lower jaw), the morphology of the roots of all premolars are comparable (no furcation involvement).

Additionally, the haptic of the plaster material could as well have influenced our results due to the difficulties of simulating realistic bone morphology and haptic. However, the presented in vitro analyses allow investigating defined and clinically not measurable parameters under reproducible conditions, thereby increasing the sensitivity of the comparisons. Last but not least, the authors want to point out that the present study focused on the evaluation of the modified training program in routine teaching procedure and not to the best possible effectiveness of removal of simulated biofilm or hard deposits of undergraduate students.

Future studies should investigate during routine students' workshops the combination of sonic scalers and curettes versus ultrasonic and subgingival air polishing with non-abrasive powder under clinical setting [10]. By teaching all these treatment methods for periodontal non-surgical therapy in dental school routine, it can be presumed that the next generation of dentists will work with the highest effectiveness concomitant with the lowest presumed number of side-effects. However, this was not evaluated in the current study. Therefore, all efforts should be done to improve the training programs and lectures in periodontology.

\section{Conclusions}

Within the limitations of the present study, it can be concluded that a systematic digitized and interactive training program in manikin heads is helpful in the training of root surface debridement in less-experienced operators. After such training, the participants are able to remove simulated biofilm with an efficacy of more than $70 \%$ in mean and nearly $80 \%$ of the hard deposits. The performance as efficacy over treatment time improved continuously for both groups of instruments. Future studies should evaluate the interaction of direct digital feedback systems and user motivation during training.

Acknowledgment The authors would like to thank José Muñoz of the Young Innovation Company (Young Innovations Europe GmbH, Heidelberg, Germany) for technical support. This study was technically (instruments provided) supported by Young Innovation Company (Young Innovations Europe GmbH, Heidelberg, Germany).

Funding information Open Access funding provided by Projekt DEAL. The work was supported by the Clinic of Conservative Dentistry and Periodontology, University of Kiel, Germany, whereas, curettes for the 
participant were provided by the Young Innovations Europe $\mathrm{GmbH}$ (Heidelberg, Germany).

\section{Compliance with ethical standards}

Conflict of interest The authors declare that they have no conflict of interest.

Ethical approval All procedures performed in studies involving human participants were in accordance with the ethical standards of the institutional and/or national research committee (local approved: D509/18) and with the 1964 Helsinki declaration and its later amendments or comparable ethical standards.

Informed consent Informed consent was obtained from all individual participants included in the study.

Open Access This article is licensed under a Creative Commons Attribution 4.0 International License, which permits use, sharing, adaptation, distribution and reproduction in any medium or format, as long as you give appropriate credit to the original author(s) and the source, provide a link to the Creative Commons licence, and indicate if changes were made. The images or other third party material in this article are included in the article's Creative Commons licence, unless indicated otherwise in a credit line to the material. If material is not included in the article's Creative Commons licence and your intended use is not permitted by statutory regulation or exceeds the permitted use, you will need to obtain permission directly from the copyright holder. To view a copy of this licence, visit http://creativecommons.org/licenses/by/4.0/.

\section{References}

1. Papapanou PN, Sanz M, Buduneli N, Dietrich T, Feres M, Fine DH, Flemmig TF, Garcia R, Giannobile WV, Graziani F, Greenwell H, Herrera D, Kao RT, Kebschull M, Kinane DF, Kirkwood KL, Kocher T, Kornman KS, Kumar PS, Loos BG, Machtei E, Meng H, Mombelli A, Needleman I, Offenbacher S, Seymour GJ, Teles R, Tonetti MS (2018) Periodontitis: Consensus report of workgroup 2 of the 2017 World Workshop on the Classification of Periodontal and Peri-Implant Diseases and Conditions. J Clin Periodontol 45(Suppl 20):S162-S170

2. Petersilka GJ, Ehmke B, Flemmig TF (2000) Antimicrobial effects of mechanical debridement. Periodontol 2002(28):56-71

3. Westfelt E (1996) Rationale of mechanical plaque control. J Clin Periodontol 23(3 Pt 2):263-267

4. Lowenberg B, Thibault J, Lawrence C, Sodek J (1986) The influence of chemically-induced modifications of root surfaces on cell migration, attachment, and orientation. J Dent Res 65(7):1010 1015

5. Frantz B, Polson A (1988) Tissue interactions with dentin specimens after demineralization using tetracycline. J Periodontol 59(11):714-721

6. Babay N (2000) Comparative SEM study on the effect of root conditioning with EDTA or tetracycline Hcl on periodontally involved root surfaces. Indian J Dent Res 11(2):53-57

7. Khosravi M, Bahrami ZS, Atabaki MSJ, Shokrgozar MA, Shokri F (2004) Comparative effectiveness of hand and ultrasonic instrumentations in root surface planing in vitro. J Clin Periodontol 31(3):160-165
8. Badersten A, Nilveus R, Egelberg J (1984) Effect of nonsurgical periodontal therapy. II. Severely advanced periodontitis. J Clin Periodontol 11(1):63-76

9. Badersten A, Nilveus R, Egelberg J (1981) Effect of nonsurgical periodontal therapy. I. Moderately advanced periodontitis. J Clin Periodontol 8(1):57-72

10. Laleman I et al (2017) Subgingival debridement: end point, methods and how often? Periodontol 75(1):189-204

11. Van der Weijden GAF, Dekkers GJ, Slot DE (2019) Success of non-surgical periodontal therapy in adult periodontitis patients: a retrospective analysis. Int J Dent Hyg 17(4):309-317

12. Rühling A et al (2002) Learning root debridement with curettes and power-driven instruments. Part I: a training program to increase effectivity. J Clin Periodontol 29(7):622-629

13. Sanz M, Meyle J (2010) Scope, competences, learning outcomes and methods of periodontal education within the undergraduate dental curriculum: a consensus report of the 1st European Workshop on Periodontal Education-position paper 2 and consensus view 2. Eur J Dent Educ 14(Suppl 1):25-33

14. Rühling A et al (2003) Learning root debridement with curettes and power-driven instruments. Part II: Clinical results following mechanical, nonsurgical therapy. J Clin Periodontol 30(7):611-615

15. Graetz C, Plaumann A, Rauschenbach S, Bielfeldt J, Dörfer CE, Schwendicke F (2016) Removal of simulated biofilm: a preclinical ergonomic comparison of instruments and operators. Clin Oral Investig 20(6):1193-1201

16. Graetz C, Schwendicke F, Plaumann A, Rauschenbach S, Springer C, Kahl M, Sälzer S, Dörfer CE (2015) Subgingival instrumentation to remove simulated plaque in vitro: influence of operators' experience and type of instrument. Clin Oral Investig 19(5):987-995

17. Tunkel J, Heinecke A, Flemmig TF (2002) A systematic review of efficacy of machine-driven and manual subgingival debridement in the treatment of chronic periodontitis. J Clin Periodontol 29(Suppl 3):72-81 discussion 90-1

18. Flemmig TF, Petersilka GJ, Mehl A, Rüdiger S, Hickel R, Klaiber B (1997) Working parameters of a sonic scaler influencing root substance removal in vitro. Clin Oral Investig 1(2):55-60

19. Flemmig TF, Petersilka GJ, Mehl A, Hickel R, Klaiber B (1998) The effect of working parameters on root substance removal using a piezoelectric ultrasonic scaler in vitro. J Clin Periodontol 25(2): $158-163$

20. Asadoorian J, Botbyl D, Goulding MJ (2015) Dental hygienists' perception of preparation and use for ultrasonic instrumentation. Int J Dent Hyg 13(1):30-41

21. König J, Rühling A, Schlemme H, Kocher T, Schwahn C, Plagmann HC (2002) Learning root debridement with curettes and power-driven instruments in vitro: the role of operator motivation and self-assessment. Eur J Dent Educ 6(4):169-175

22. Eichenberger M, Perrin P, Neuhaus KW, Bringolf U, Lussi A (2011) Influence of loupes and age on the near visual acuity of practicing dentists. J Biomed Opt 16(3):035003

23. Dadwal A, Kaur R, Jindal V, Jain A, Mahajan A, Goel A (2018) Comparative evaluation of manual scaling and root planing with or without magnification loupes using scanning electron microscope: a pilot study. J Indian Soc Periodontol 22(4):317-321

24. Corbella S, Taschieri S, Cavalli N, Francetti L (2018) Comparative evaluation of the use of magnification loupes in supragingival scaling procedures. J Investig Clin Dent 9(2):e12315

25. Sunell S, Rucker L (2004) Surgical magnification in dental hygiene practice. Int J Dent Hyg 2(1):26-35

26. Hayes MJ, Taylor JA, Smith DR (2016) Introducing loupes to clinical practice: dental hygienists experiences and opinions. Int J Dent Hyg 14(3):226-230

27. Graetz C, Schorr S, Christofzik D, Dörfer CE, Sälzer S (2020) How to train periodontal endoscopy? Results of a pilot study removing simulated hard deposits in vitro. Clin Oral Investig 24(2):607-617 
28. Graetz C, Plaumann A, Wittich R, Springer C, Kahl M, Dörfer CE, el-Sayed KF (2017) Removal of simulated biofilm: an evaluation of the effect on root surfaces roughness after scaling. Clin Oral Investig 21(4):1021-1028

29. Kocher T, Ruhling A, Momsen H, Plagmann HC (1997) Effectiveness of subgingival instrumentation with power-driven instruments in the hands of experienced and inexperienced operators. A study on manikins. J Clin Periodontol 24(7):498-504

30. Auplish G, Needleman IG, Moles DR, Newman HN (2000) Diamond-coated sonic tips are more efficient for open debridement of molar furcations. A comparative manikin study. J Clin Periodontol 27(5):302-307

31. Kumar P, Das SJ, Sonowal ST, Chawla J (2015) Comparison of root surface roughness produced by hand instruments and ultrasonic scalers: an invitro study. J Clin Diagn Res 9(11):ZC56-ZC60

32. Aspriello SD, Piemontese M, Levrini L, Sauro S (2011) Ultramorphology of the root surface subsequent to handultrasonic simultaneous instrumentation during non-surgical periodontal treatments: an in vitro study. J Appl Oral Sci 19(1):74-81
33. Zappa U, Smith B, Simona C, Graf H, Case D, Kim W (1991) Root substance removal by scaling and root planing. J Periodontol 62(12):750-754

34. Flemmig TF, Petersilka GJ, Mehl A, Hickel R, Klaiber B (1998) Working parameters of a magnetostrictive ultrasonic scaler influencing root substance removal in vitro. J Periodontol 69(5): $547-553$

35. Gankerseer EJ, Walmsley AD (1987) Preliminary investigation into the performance of a sonic scaler. J Periodontol 58(11):780-784

36. Sugaya T, Kawanami M, Kato H (2002) Accessibility of an ultrasonic furcation tip to furcation areas of mandibular first and second molars. J Int Acad Periodontol 4(4):132-137

37. Canakci V et al (2004) Effect of handedness on learning subgingival scaling with curettes: a study on manikins. Int J Neurosci 114(11):1463-1482

Publisher's note Springer Nature remains neutral with regard to jurisdictional claims in published maps and institutional affiliations. 